\title{
(D620N) VPS35 causes the impairment of Wnt/ $\beta$-catenin signaling cascade and mitochondrial dysfunction in a PARK17 knockin mouse model
}

Ching-Chi Chiu 1,2,3,4, Yi-Hsin Weng ${ }^{1,4,5,6}$, Ying-Zu Huang ${ }^{1,4,5,6,7}$, Rou-Shayn Chen ${ }^{1,4,5,6}$, Yu-Chuan Liu ${ }^{8}$, Tu-Hsueh Yeh (1) ${ }^{9}$, Chin-Song Lu, ${ }^{1,4}$, Yan-Wei Lin ${ }^{1}$, Yu-Jie Chen ${ }^{1}$, Chia-Chen Hsu' ${ }^{1}$ Chi-Han Chiu', Yu-Ting Wang ${ }^{1}$, Wan-Shia Chen ${ }^{10}$, Shu-Yu Liu' ${ }^{10}$ and Hung-Li Wang ${ }^{1,4,5,10}$

\begin{abstract}
Patients with familial type 17 of Parkinson's disease (PARK17) manifest autosomal dominant pattern and late-onset parkinsonian syndromes. Heterozygous (D620N) mutation of vacuolar protein sorting 35 (VPS35) is genetic cause of PARK17. We prepared heterozygous VPS35 ${ }^{\text {D620N/+ }}$ knockin mouse, which is an ideal animal model of (D620N) VPS35induced autosomal dominant PARK17. Late-onset loss of substantia nigra pars compacta (SNpc) dopaminergic (DAergic) neurons and motor deficits of Parkinson's disease were found in 16-month-old VPS35 $5^{\mathrm{D} 20 \mathrm{~N} /+}$ mice. Normal function of VPS35-containing retromer is needed for activity of Wnt/ $\beta$-catenin cascade, which participates in protection and survival of SNpc DAergic neurons. It was hypothesized that (D620N) VPS35 mutation causes the malfunction of VPS35 and resulting impaired activity of Wnt/ $\beta$-catenin pathway. Protein levels of Wnt1 and nuclear $\beta$-catenin were reduced in SN of 16-month-old VPS35 ${ }^{\mathrm{D} 20 \mathrm{~N} /+}$ - knockin mice. Downregulated protein expression of survivin, which is a target gene of nuclear $\beta$-catenin, and upregulated protein levels of active caspase- 8 and active caspase-9 were observed in SN of VPS35 $5^{\mathrm{D} 620 \mathrm{~N} /+}$ mice at age of 16 months. VPS35 is involved in controlling morphology and function of mitochondria. Impaired function of VPS35 caused by (D620N) mutation could lead to abnormal morphology and malfunction of mitochondria. A significant decrease in mitochondrial size and resulting mitochondrial fragmentation was found in tyrosine hydroxylase-positive and neuromelanin-positive SNpc DAergic neurons of 16-month-old VPS35 $5^{\mathrm{D} 20 \mathrm{~N} /+}$ mice. Mitochondrial complex I activity or complex IV activity was reduced in SN of 16-month-old VPS35 $5^{\mathrm{D} 620 \mathrm{~N} /+}$ mice. Increased level of mitochondrial ROS and oxidative stress were found in SN of 16-month-old VPS35 ${ }^{\mathrm{D} 620 \mathrm{~N} /+}$ mice. Levels of cytosolic cytochrome $\mathrm{c}$ and active caspase-3 were increased in SN of VPS35 $5^{\text {D620N/+ }}$ mice aged 16 months. Our results suggest that PARK17 mutant (D620N) VPS35 impairs activity of Wnt/ $\beta$-catenin signaling pathway and causes abnormal morphology and dysfunction of mitochondria, which could lead to neurodegeneration of SNpc DAergic cells.
\end{abstract}

Correspondence: Hung-Li Wang (hlwns@mail.cgu.edu.tw)

${ }^{1}$ Neuroscience Research Center, Chang Gung Memorial Hospital at Linkou, Taoyuan, Taiwan

2Department of Nursing, Chang Gung University of Science and Technology, Taoyuan, Taiwan

Full list of author information is available at the end of the article These author contributed equally: Ching-Chi Chiu, Yi-Hsin Weng Edited by P. G. Mastroberardino

\section{Introduction}

About $1 \%$ of the population older than the age of 60 are affected with Parkinson's disease (PD), which is the most prevalent neurodegenerative motor disease ${ }^{1}$. PD patients exhibit classical symptoms of motor dysfunction, which include akinesia, bradykinesia, resting tremor, and rigidity. Neuropathological hallmarks underlying PD clinical manifestations are neurodegeneration of substantia nigra

\section{(c) The Author(s) 2020}

(c) Open Access This article is licensed under a Creative Commons Attribution 4.0 International License, which permits use, sharing, adaptation, distribution and reproduction cc) in any medium or format, as long as you give appropriate credit to the original author(s) and the source, provide a link to the Creative Commons license, and indicate if changes were made. The images or other third party material in this article are included in the article's Creative Commons license, unless indicated otherwise in a credit line to the material. If material is not included in the article's Creative Commons license and your intended use is not permitted by statutory regulation or exceeds the permitted use, you will need to obtain permission directly from the copyright holder. To view a copy of this license, visit http://creativecommons.org/licenses/by/4.0/. 
pars compacta (SNpc) dopaminergic (DAergic) cells and presence of intracellular Lewy bodies ${ }^{2}$. Most of PD patients $(\sim 90 \%)$ are sporadic cases, and hereditary genetic mutations cause $\sim 10 \%$ of PD cases ${ }^{1,3-6}$. Patients with familial type 17 of Parkinson's disease (PARK17) manifest autosomal dominant pattern and late-onset parkinsonism syndromes $^{3,4,6}$. In 2011, two research groups ${ }^{7,8}$ used exome sequencing technology to identify mutations of PARK17 in vacuolar protein sorting 35 (VPS35) gene. Heterozygous Asp620Asn (D620N) mutation of VPS35 is a confirmed genetic cause of PARK17 (refs. ${ }^{7-10}$ ). PARK17 patients with (D620N) VPS35 mutation displayed cardinal symptoms of PD and exhibited the improvement after Ldopa treatment ${ }^{7,8}$. Interestingly, (D620N) mutation of VPS35 was also observed in sporadic PD patients ${ }^{11}$. Knockin mouse harboring disease-causing gene mutation is an ideal animal model for investigating the pathogenic mechanism of hereditary human disease ${ }^{12}$. Knockin mouse expressing PARK17 (D620N) VPS35 has not been used to study molecular pathogenic mechanisms involved in (D620N) VPS35 mutation-induced loss of SNpc DAergic cells and resulting PD.

VPS35 protein is expressed in various brain areas, such as striatum (ST) and $\mathrm{SN}^{13}$. VPS35 is the critical element of retromer multi-subunit complex ${ }^{14-18}$. Retromer is consisted of VPS26-VPS29-VPS35 trimer and sorting nexin (SNX) dimer. Retromer complex controls intracellular trafficking of proteins by associating with endosomes and mediating protein transport from endosomes to transGolgi network (TGN) or plasma membrane ${ }^{14-16,18}$. SNX dimer is required for recruiting retromer to endosomes. VPS35-VPS29-VPS26 trimer participates in cargo binding and functions as the cargo recognition complex ${ }^{14,18}$. VPS35-containing retromer complex-mediated protein trafficking regulates activity of several signal transduction pathways, including Wnt signaling cascades $^{10,17,19}$. Wntless promotes Wnt secretion by transporting Wnt proteins from TGN to cell membrane where Wnt ligands are released. VPS35-containing retromer, which mediates the endosome-to-TGN trafficking of Wntless, is required for normal release of Wnt proteins and subsequent activation of Wnt signaling pathways ${ }^{19-23}$. One of Wnt signaling cascades is canonical Wnt/ $\beta$-catenin pathway ${ }^{24-26}$. Wnt protein plays key roles in development and survival of SNpc DAergic neurons through activating Wnt/ $\beta$-catenin signaling cascade ${ }^{27,28}$. Wnt receptor Frizzled-1 and $\beta$-catenin are found in SNpc DAergic neurons of mouse brain ${ }^{29}$. Wnt1 promotes differentiation of midbrain DAergic neurons and maintains survival of $\mathrm{SN}$ DAergic neurons by activating $\mathrm{Wnt} / \beta$-catenin signaling cascade $^{30,31}$. Wnt1 activation of $\mathrm{Wnt} / \beta$-catenin cascade prevents 6-hydroxydopamine-induced neurotoxic effect on DAergic neurons ${ }^{32}$. Specific knockout of $\beta$-catenin causes the loss of SNpc DAergic neurons, indicating that $\beta$-catenin is needed for survival of SNpc DAergic cells ${ }^{33}$. (D620N) VPS35 mutation has been shown to disrupt cargo sorting function of retromer and cause trafficking defects $^{34}$. Therefore, it is very likely that PARK17 mutant (D620N) VPS35 causes a defective secretion of Wnt and an impaired activity of $\mathrm{Wnt} / \beta$-catenin signaling, leading to cell death of SNpc DAergic neurons and PD ${ }^{25,35}$.

Mitochondria play an essential role in ATP generation, regulation of intracellular calcium level, activation of apoptotic pathway, and oxidative stress response ${ }^{36,37}$. Mitochondrial dysfunction is a major pathogenic mechanism that causes neuronal death of SNpc DAergic cells, and resulting sporadic or familial $\mathrm{PD}^{36-38}$. Malfunction of mitochondria is believed to cause an increase in mitochondrial level of ROS and cytochrome $\mathrm{c}$ release to cytosol, which leads to induction of mitochondriamediated apoptotic pathway and loss of SNpc DAergic neurons in PD patients ${ }^{36,37}$. Normal morphology and function of mitochondria is maintained by constant, and dynamic process of fission and fusion ${ }^{36,37}$. Abnormal morphology of mitochondria caused by an impaired process of mitochondrial dynamics results in mitochondrial dysfunction, overproduction of ROS, and subsequent apoptotic death of SNpc DAergic neurons ${ }^{36,37}$. VPS35 deficiency has been shown to cause fragmentation of mitochondria in DAergic neurons by impairing mitochondrial fusion $^{39}$. A previous in vitro study reported that mutant (D620N) VPS35-induced mitochondrial fragmentation and dysfunction by promoting fission process of mitochondria ${ }^{40}$. Further study using (D620N) VPS35 knockin mouse is required to provide in vivo evidence that PARK17 mutant (D620N) VPS35 causes abnormal morphology and malfunction of mitochondria.

Heterozygous (D620N) mutation of VPS35 causes autosomal dominant PARK17 (refs. ${ }^{7-10}$ ). In the present study, heterozygous VPS35 $5^{\mathrm{D} 620 \mathrm{~N} /+}$ knockin mice were prepared as the animal model of mutant (D620N) VPS35induced autosomal dominant PARK17. Heterozygous VPS35 $5^{\mathrm{D} 620 \mathrm{~N} /+}$ mice displayed late-onset pathological and behavioral phenotypes of PD. Our data suggest that PARK17 mutant (D620N) VPS35 causes cell death of SNpc DAergic neurons via impairing activity of Wnt/ $\beta$-catenin neuroprotective signaling, and causing abnormal morphology and dysfunction of mitochondria.

\section{Materials and methods}

\section{Preparation of heterozygous VPS $35^{\mathrm{D} 620 \mathrm{~N} /+}$ knockin mice}

GAT codon for Asp-620 in exon 15 of mouse VPS35 gene was altered to AAT codon of Asn by performing oligonucleotide-directed mutagenesis using PCR amplification. DNA fragment of exon 15 containing (D620N) mutation was ligated into pBluescript vector. LoxP-flanked neomycin selection (Neo) sequence was added into intron 15. Knockin target vector containing (D620N) fragment of 
exon 15, LoxP-flanked Neo cassette, $5^{\prime}$ and $3^{\prime}$ homologous sequence was transfected into $129 / \mathrm{Sv}$ ES cells. PCR assays were conducted to screen neomycin-resistant colonies with correct homologous recombination. ES cells with correct targeting were microinjected into C57BL/6 J blastocysts. Then, chimeric mouse was crossed with wild-type (WT) C57BL/6 J mice. Resulting mutant heterozygous F1 mice were tested for germline transmission by PCR assay. (D620N) VPS35 knockin mutation was confirmed by direct sequencing of exon 15 after PCR amplification of tail DNA. Neo cassette was removed by breeding F1 mice carrying (D620N) VPS35 mutation with EllA-Cre transgenic mice. Heterozygous VPS $35^{\mathrm{D} 620 \mathrm{~N} /+}$ knockin mice were maintained on C57BL/6 J background. Procedures of animal experiments were approved by Institutional Animal Care and Use Committee (IACUC No. CGU107-166) of Chang Gung University.

\section{PCR assays of knockin mice expressing mutant (D620N) VPS35}

Forward primer (5'-TCTATGTATACCAGGCATTTT CTCTATATG- $3^{\prime}$ ) and reverse primer (5'-AAAG ACAACAAGAAGAAATTAGATATCCTG-3') were used to perform PCR assay using tail DNA of WT or VPS35 $5^{\mathrm{D} 620 \mathrm{~N} /+}$ mouse. PCR products for WT allele and VPS35 $^{\mathrm{D} 620 \mathrm{~N}}$ allele were 660 and $710 \mathrm{bp}$, respectively.

RT-PCR confirmation of (D620N) VPS35 knockin mutation

Total RNA was purified from SN of WT or VPS35 $5^{\mathrm{D} 620 \mathrm{~N} /+}$ mouse by using Trizol Reagent (Invitrogen). The first strand cDNA was synthesized using total RNA extracted from SN of WT or VPS35 $5^{\text {D620N/+ }}$ mouse, as described previously ${ }^{41}$. Then, PCR was carried out with cDNA and specific primers of exon 15 of VPS35 gene. PCR product was then gel purified and used for DNA sequencing.

\section{Behavioral tests}

Following behavioral tests were performed to assess motor performance of WT or VPS35 $5^{\mathrm{D} 620 \mathrm{~N} /+}$ mice: (1) to measure distance and velocity of locomotion, mice were placed in an open-field behavioral space. Video camera was used to record locomotion of mice for $40 \mathrm{~min}$, which was then quantified using TopScan (Clever Sys.) motion tracking software. (2) Motor performance of WT or VPS35 $5^{\mathrm{D} 620 \mathrm{~N} /+}$ mice was determined by conducting pole test. For this test, mice were placed on top of a pole, and the base of pole was put in home cage. Mice descend along the pole and walk to home cage. Following 2 days of training, mice carried out five trials, and the time needed for walking along the pore was recorded.

\section{Immunohistochemical staining}

Following the anesthesia, WT or VPS35 $5^{\mathrm{D} 620 \mathrm{~N} /+}$ mice were perfused with $4 \%$ paraformaldehyde. Permeabilized coronal sections $(20 \mu \mathrm{m})$ of frozen brain were interacted with one of following primary antibodies: (1) tyrosine hydroxylase (TH) antibody (Merck Millipore, MAB318); (2) NeuN antiserum (Merck Millipore, MAB377); (3) $\alpha$-synuclein antibody (Proteintech, 10842-1-AP); (4) phospho- $\alpha$-synuclein ${ }^{\text {Ser129 }}$ antiserum (Abcam, ab51253); and (5) phospho-Tau ${ }^{\text {Ser202/Thr205 }}$ antiserum (Thermo Scientific, MN1020). Following the incubation with biotinylated secondary antibody, brain slices were incubated with avidin-biotin-HRP (Vector Laboratories). As described previously ${ }^{41}$, StereoInvestigator software (MBF Bioscience) was used to quantify the number of $\mathrm{TH}$ positive SN DAergic neurons or NeuN-positive neurons. ImageJ software was utilized to measure optical density of striatal $\mathrm{TH}$ staining.

\section{Immunoblotting study}

As described in our previous study ${ }^{41}$, $\mathrm{SN}$ tissue was dissected out from coronal brain slices of midbrain under a microscope according to the stereotaxic atlas of mouse brain. CHAPS lysis buffer was used to obtain proteins from SN tissue of WT or VPS35 $5^{\mathrm{D} 620 \mathrm{~N} /+}$ mouse. For preparing cytosolic fraction, SN tissues were sonicated in CHAPS buffer containing protease inhibitor cocktail (Sigma). After centrifugation at 12,000 r.p.m., the supernatant, which contains cytosolic fraction, was obtained and used for immunoblotting study. According to manufacturer's protocol, Mitochondria Isolation Kit (ThermoFisher Scientific) was utilized to isolate mitochondrial proteins from $\mathrm{SN}$ tissues of WT or VPS35 $5^{\mathrm{D} 620 \mathrm{~N} /+}$ mouse. To collect nuclear fraction, $\mathrm{SN}$ tissues were sonicated in lysis buffer $(5 \mathrm{mM}$ DTT, $2 \mathrm{mM} \mathrm{MgCl} 2,10 \mathrm{mM}$ Tris, $\mathrm{pH}=7.4$, and commercial proteinase inhibitor), and homogenates were centrifuged at 2500 r.p.m. Resulting pellet was resuspended in lysis buffer and centrifuged at 2500 r.p.m. Nuclear extract was collected by adding extraction buffer (10\% glycerol, $1 \mathrm{mM}$ DTT, $2 \mathrm{mM} \mathrm{MgCl}_{2}, 2 \mathrm{mM}$ EDTA, $0.4 \mathrm{M} \mathrm{KCl}, 20 \mathrm{mM}$ Tris, $\mathrm{pH}=$ 8.0 and commercial proteinase inhibitor) to the pellet. Following centrifugation at 15,000 r.p.m., supernatant was utilized as nuclear extract.

Cytosolic, mitochondrial, or nuclear fractions of $\mathrm{SN}$ or protein extracts of SN $(30 \mu \mathrm{g})$ separated on SDS-PAGE were transferred to nitrocellulose membranes. Then, membranes were hybridized with following primary antibodies: (1) VPS35 antibody (Abcam, ab10099); (2) $\alpha$ synuclein antiserum (Proteintech, \#10842-1-AP); (3) phospho- $\alpha$-synuclein ${ }^{\text {Ser129 }}$ antibody (Abcam, ab51253);

(4) Tau antiserum (Santa Cruz Biotechnology, sc-32274); (5) phospho-Tau ${ }^{\text {Ser202,/Thr205 }}$ antiserum (Thermo Scientific, MN1020); (6) Wnt1 antibody (Abcam, ab15251); (7) $\beta$-catenin antiserum (Abcam, ab6302); (8) survivin antibody (Abcam, ab76424); (9) cytochrome c antiserum (Abcam, ab133504); (10) cleaved caspase-8 antibody (Cell Signaling Technology, \#9496, iREAL Biotechnology, IR99409); (11) cleaved caspase-9 antiserum (Cell Signaling 
Technology, \#20750); (12) cleaved caspase-3 antibody (Cell Signaling Technology, \#9662); (13) mitofusin 2 antiserum (Cell Signaling Technology, \#9482); (14) Drp1 antibody (Cell Signaling Technology, \#8570); (15) actin antiserum (Abcam, ab8226); (16) histone H3 antibody (Merck, 05-449); and (17) COX IV antiserum (Abcam, ab16056). Immunoreactive proteins on membranes were visualized by interacting with appropriate HRPconjugated secondary antibody.

\section{Transmission electron microscopy}

The brains of mice were fixed with $4 \%$ paraformaldehyde/ $2.5 \%$ glutaraldehyde reagent. SN sections were cut in 1$\mathrm{mm}^{3}$ slices and incubated in $1 \%$ osmium tetroxide for $2 \mathrm{~h}$. SN slices were dehydrated with an increasing concentrations of ethanol solutions. Dehydrated SN slices were then embedded in Epon resin (Electron Microscopy Sciences) and sectioned into ultrathin specimens $(80 \mathrm{~nm})$. Cellular and mitochondrial structures were visualized using transmission electron microscope (Hitachi HT7800). The mitochondrial size was quantified by using ImageJ software.

\section{Analysis of mitochondrial complex activity}

To measure mitochondrial complex I activity, $30 \mu \mathrm{g}$ mitochondrial extracts were loaded into 96-well microplate and interacted with anti-complex I monoclonal antibody coated in the microplate. The oxidation of reduced NADH to $\mathrm{NAD}^{+}$caused by mitochondrial complex I leads to an increase in the absorbance at OD $450 \mathrm{~nm}$.

For measuring activity of mitochondrial complex II, mitochondrial fraction $(30 \mu \mathrm{g})$ was added into microplate wells coated with anti-complex II antibody. Mitochondrial complex II catalyzes ubiquinone to ubiquinol, which couples DCPIP dye (blue color) to DCPIPH2 (colorless). Mitochondrial complex II activity was examined by a decreased OD $600 \mathrm{~nm}$ value.

To measure mitochondrial complex III activity, mitochondrial extracts $(30 \mu \mathrm{g})$ were incubated with complex III activity solution containing oxidized cytochrome c. Mitochondrial complex III converts oxidized cytochrome $\mathrm{c}$ to reduced cytochrome $\mathrm{c}$, leading to an increase at OD $550 \mathrm{~nm}$.

For measuring activity of mitochondrial complex IV, $30 \mu \mathrm{g}$ of mitochondrial fraction was added into microplate coated with anti-complex IV monoclonal antibody. Mitochondrial complex IV activity was evaluated by oxidation of reduced cytochrome $\mathrm{c}$, which resulted in a decrease at OD $550 \mathrm{~nm}$.

\section{Measurement of intracellular ATP}

ATP Colorimetric/Fluorometric Assay Kit (BioVision) was utilized to examine the content of intracellular ATP. Mitochondrial extract $(20 \mu \mathrm{l})$ was added into 96-well plate and interacted with reaction mix of kit. After incubation for $30 \mathrm{~min}$, intracellular ATP level was colorimetrically determined by measuring OD $570 \mathrm{~nm}$.

\section{Measurement of mitochondrial respiration}

SN tissues of WT or VPS35 $5^{\mathrm{D} 620 \mathrm{~N} /+}$ mice were loaded into XFe24 microplate (Agilent). Then, mitochondrial respiration was determined by measuring mitochondrial oxygen consumption rate with aid of Seahorse XFe24 Analyzer (Agilent).

\section{Analysis of ROS}

The level of ROS was evaluated by utilizing OxiSelect In Vitro ROS/RNS assay kit (Cell Biolabs). A total of $50 \mu \mathrm{l}$ of mitochondria extracts were applied into microplate and interacted with catalyst reagent $(50 \mu \mathrm{l})$ of kit. DCFHDiOxyQ, the ROS probe, was added into mixture and oxidized to fluorescent DCF in the presence of ROS. Microplate detector was utilized to determine fluorescent intensity of DCF at $480 \mathrm{~nm}$ (excitation)/530 nm (emission).

\section{Determination of mitochondrial lipid peroxidation}

TBARS (thiobarbituric acid reactive substances) assay kit (Cayman Chemicals) was utilized to evaluate mitochondrial lipid peroxidation of SN tissue from WT or VPS35 $5^{\mathrm{D} 620 \mathrm{~N} /+}$ mouse. Thiobarbituric acid binds to malondialdehyde, final metabolite of lipid peroxidation, and generates thiobarbituric acid-malondialdehyde adduct. Mitochondrial fractions or malondialdehyde standards were incubated with thiobarbituric acid, and the level of malondialdehyde was then examined by measuring OD $540 \mathrm{~nm}$.

\section{Colorimetric determination of caspase- 9 or caspase- 3 activity}

Colorimetric caspase- 9 or caspase- 3 assay kit (Abcam) was used to analyze the activity of caspase- 9 or caspase- 3 in SN of WT or VPS35 $5^{\mathrm{D} 620 \mathrm{~N} /+}$ mice. Active caspase-9 and active caspase-3 recognize the LEHD and DEVD sequence, respectively. Active caspase cleaves chromophore $p$-nitroaniline- or $p$-nitroanilide-labeled substrates, and releases chromophore $p$-nitroaniline or $p$-nitroanilide. The activity of caspase- 9 or caspase- 3 was evaluated by determining OD $400 \mathrm{~nm}$.

\section{Statistics}

Unpaired Student's $t$ test was utilized to assess statistical significance between two groups. One-way ANOVA with Tukey test was utilized to analyze multiple groups. The $P$ value $<0.05$ was considered as significant.

\section{Results}

Heterozygous VPS35 $5^{\mathrm{D} 20 \mathrm{~N} /+}$ knockin mice exhibit lateonset loss of SNpc DAergic neurons

Heterozygous (D620N) mutation of VPS35 is a confirmed genetic cause of autosomal dominant PARK17 
$\left(\right.$ refs. $\left.{ }^{7-10}\right)$. Therefore, we generated heterozygous VPS35 ${ }^{\mathrm{D} 620 \mathrm{~N} /+}$ knockin mice (Fig. 1A) as mouse model of mutant (D620N) VPS35-induced autosomal dominant PARK17. VPS35 ${ }^{\mathrm{D} 620 \mathrm{~N} /+}$ knockin mouse was prepared by performing target vector-mediated homologous recombination and introducing (D620N; GAT $\rightarrow$ AAT) mutation in exon 15 of VPS35 gene. Total RNA was extracted from SN of WT or VPS35 ${ }^{\mathrm{D} 620 \mathrm{~N} /+}$ knockin mice. Subsequent RT-PCR reaction and direct DNA sequencing were performed to verify heterozygous (D620N) mutation of VPS35 mRNA in VPS35 $5^{\text {D20N/+ }}$ knockin mouse (Fig. 1B). PCR reactions using genomic DNA of WT or VPS3 $5^{\text {D620N/+ }}$ mouse were conducted to obtain DNA fragments of VPS35 exons and their adjacent introns. Subsequent DNA sequencing indicated that except $(\mathrm{D} 620 \mathrm{~N}$; GAT $\rightarrow$ AAT) mutation in exon 15, sequences of exons and adjacent introns of VPS35 gene in VPS35 $5^{\text {D620N/+ }}$ knockin mouse were not altered (Supplementary Table 1 and Supplementary Fig. 1). RT-PCR analysis showed that a similar level of mRNA, which encodes full-length coding region of VPS35 mRNA, was found in SN of WT or $\mathrm{VPS}^{2} 5^{\mathrm{D} 620 \mathrm{~N} /+}$ mice (Fig. $1 \mathrm{C} ; n=4$ mice). Real-time quantitative RT-PCR assays also demonstrated that VPS35 mRNA level in SN of VPS35 $5^{\text {D620N/+ }}$ knockin mice was similar to that of WT mice (Fig. 1C). Immunoblotting assays indicated that a similar expression of VPS35 protein was found in SN of WT or VPS35 $5^{\mathrm{D} 620 \mathrm{~N} /+}$ mice (Fig. 1D).
Breeding with VPS35 ${ }^{\mathrm{D} 620 \mathrm{~N} /+}$ mice produced WT mice, heterozygous knockin mice and homozygous knockin mice with an expected Mendelian ratio. Similar to WT mice, heterozygous VPS35 $5^{\mathrm{D} 620 \mathrm{~N} /+}$ mice exhibited normal body weight and survival rate (Fig. 1E, F).

We performed immunohistochemical staining of $\mathrm{TH}$ positive SNpc DAergic neurons using WT or VPS35 $5^{\mathrm{D} 620 \mathrm{~N} /}$ ${ }^{+}$mice aged 12 or 16 months. Number of TH-positive SNpc DAergic neurons of 12-month-old VPS35 ${ }^{\mathrm{D} 620 \mathrm{~N} /+}$ knockin mice was similar to that of age-matched WT mice (Fig. 2A). Consistent with previous studies demonstrating that heterozygous (D620N) VPS35 mutation causes late-onset $\mathrm{PD}^{7,8}$, heterozygous $\mathrm{VPS}^{\mathrm{D}} 5^{\mathrm{D} 20 \mathrm{~N} /+}$ mice at age of 16 months display a decrease in number of $\mathrm{TH}$-positive SNpc DAergic neurons (Fig. 2A). Number of Nissl ${ }^{+}$cells was significantly decreased in SNpc of VPS3 $5^{\mathrm{D} 620 \mathrm{~N} /+}$ knock mice aged 16 months (Fig. 2B). Loss of SNpc DAergic neurons observed in VPS35 $5^{\mathrm{D} 620 \mathrm{~N} /+}$ mice aged 16 months should result in reduction of DAergic nigrostriatal terminals. Compared to WT mice at age of 16 months, level of striatal $\mathrm{TH}$ immunoreactivity was decreased in agematched VPS35 ${ }^{\mathrm{D} 620 \mathrm{~N} /+}$ knockin mice (Fig. 2C). In accordance with results of $\mathrm{TH}$ immunohistochemical staining, protein expression of $\mathrm{TH}$ was downregulated in $\mathrm{SN}$ and $\mathrm{ST}$ of 16-month-old VPS35 $5^{\mathrm{D} 620 \mathrm{~N} /+}$ mice (Fig. 2D). Immunocytochemical staining of neuronal marker NeuN indicated that reduction of neuronal number was not observed in the
(A)

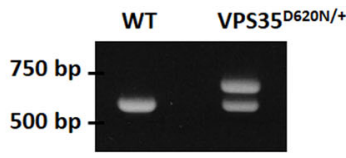

(B)

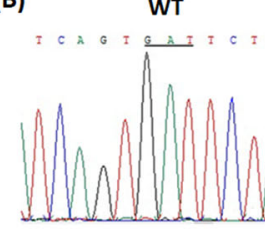

VPS35 $0620 \mathrm{~N} /+$
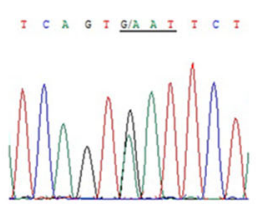

(c)

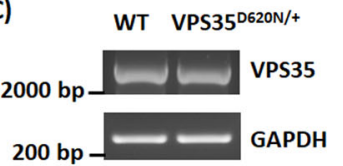

(E)

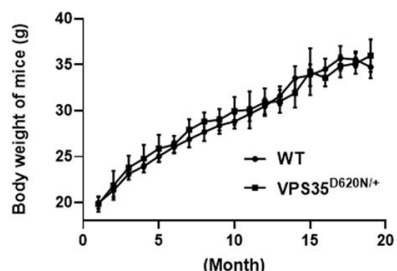

(F)

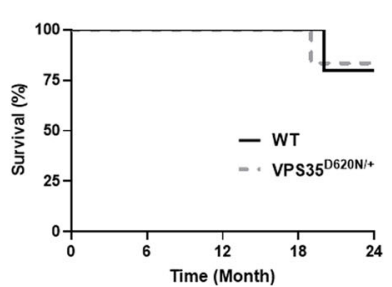

Fig. 1 Protein expression of VPS35 is not altered in SN of heterozygous VPS35 $5^{\mathrm{D} 620 \mathrm{~N} /+}$ knockin mouse. A Heterozygous VPS35 $5^{\mathrm{D} 620 \mathrm{~N} /+}$ mouse was identified by performing PCR assays using tail DNA of wild-type (WT) or VPS35 ${ }^{\mathrm{D} 620 \mathrm{~N} /+}$ mouse. Expected sizes of PCR products for WT allele and VPS35 ${ }^{\mathrm{D} 620 \mathrm{~N}}$ allele were 660 and $710 \mathrm{bp}$, respectively. B Following RT-PCR reaction using total RNA extracted from SN of WT or VPS35 $5^{\mathrm{D} 620 \mathrm{~N} /+}$ mouse, DNA sequencing showed that in contrast to normal GAT (D620) sequence of VPS35 in WT mouse, both mutated AAT (N620) and wild-type GAT (D620) sequences of VPS35 were found in heterozygous VPS35 ${ }^{\text {D620N/+ }}$ mouse. C Following RT-PCR reaction, a similar level of mRNA, which encodes full-length coding region of VPS35 mRNA, was observed in SN of WT or VPS35 ${ }^{\mathrm{D} 620 N /+}$ mouse. Real-time quantitative RT-PCR assays showed that VPS35 mRNA level in SN of VPS35 ${ }^{\mathrm{D} 620 N /+}$ knockin mice was not significantly different from that of WT mice. Each bar shows mean $\pm \mathrm{S}$. E. value of four mice. D Protein level of VPS35 in SN of VPS35 ${ }^{\mathrm{D} 620 \mathrm{~N} /+}$ knockin mouse was similar to that of VPS35 in SN of WT mouse. Each bar represents mean \pm S. E. value of five mice. E WT and VPS35 ${ }^{\mathrm{D} 620 \mathrm{~N} /+}$ mice exhibited similar body weight-age curves. Each point shows mean \pm S. E. value of 30-35 mice. F WT mice and heterozygous VPS35 D620N/+ mice displayed similar Kaplan-Meier survival curves (WT mice, $n=30 ; V^{2} 535^{D 620 N /+}$ mice, $n=30$ ). 
(A)

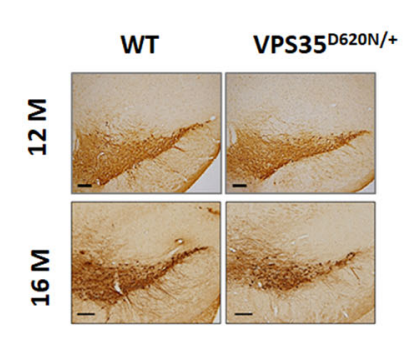

(C)

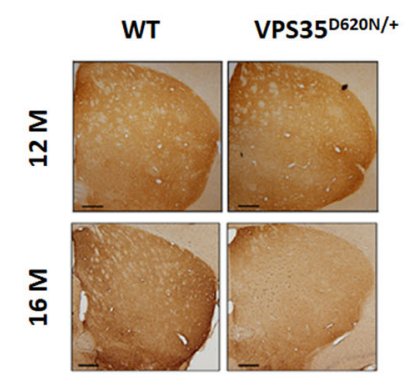

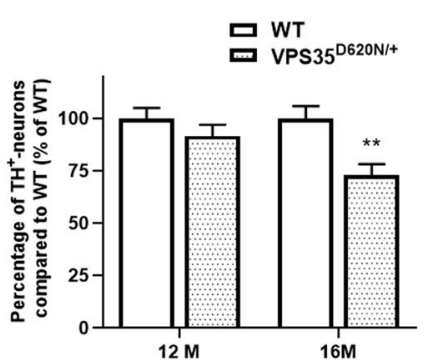

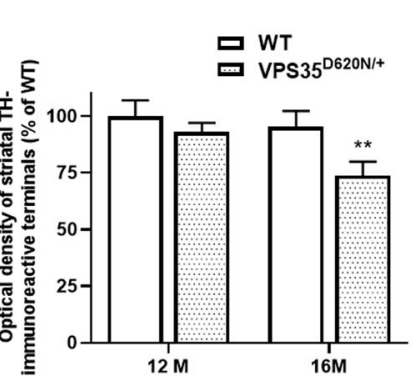

(B)

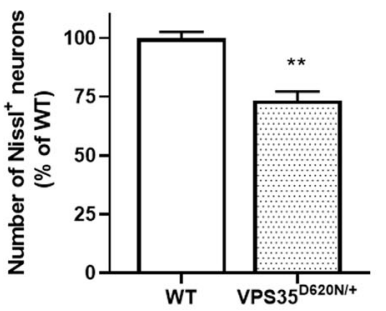

(D) SN

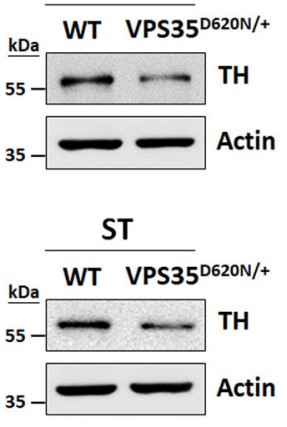

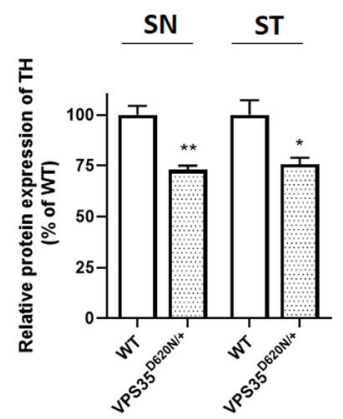

Fig. 2 Sixteen-month-old heterozygous VPS35 $5^{\mathrm{D} 620 \mathrm{~N} /+}$ knockin mice exhibit late-onset cell death of SNpc DAergic neurons and degeneration of DAergic nigrostriatal terminals. A TH staining showed that 12-month-old VPS35 ${ }^{\mathrm{D} 620 \mathrm{~N} / 4}$ knockin mice did not display a decrease in number of SNpc DAergic neurons. Compared to WT mice at age of 16 months, age-matched heterozygous VPS35 ${ }^{\mathrm{D} 620 \mathrm{~N} /+}$ mice exhibited the reduction in number of SNpc DAergic neurons. Each bar represents mean \pm S. E. value of 11 mice. ${ }^{* *} P<0.01$ compared with WT mice. B Sixteenmonth-old VPS35 ${ }^{\mathrm{D} 620 \mathrm{~N} /+}$ mice exhibited a reduction in number of Niss ${ }^{+}$cells in SNpc. Each bar shows mean \pm S.E. value of eight mice. C Compared to WT mice aged 16 months, age-matched VPS35 $5^{\mathrm{D} 620 \mathrm{~N} /+}$ knockin mice exhibited the reduction in density of striatal TH immunoreactivity. Each bar represents mean \pm S.E. value of 11 mice. D Protein level of TH was significantly decreased in SN and striatum (ST) of VPS35 ${ }^{\text {D620N/+ }}$ knockin mice at age of 16 months. Each bar shows mean \pm S.E. value of four mice. ${ }^{*} P<0.05$ compared with WT mice.

ST or cerebral cortex of 16-month-old heterozygous VPS35 ${ }^{\mathrm{D} 620 \mathrm{~N} /+}$ mice (data not shown; $n=5$ mice).

\section{Heterozygous VPS35 $5^{\mathrm{D} 20 \mathrm{~N} /+}$ knockin mice exhibit late- onset motor impairments of PD}

Mutant (D620N) VPS35-induced loss of SNpc DAergic neurons and impaired DAergic neurotransmission in the ST is expected to cause motor dysfunction, and resulting parkinsonism behavioral phenotypes, including hypokinesia and bradykinesia, of heterozygous VPS35 ${ }^{\text {D620N/+ }}$ mice. The locomotor activity of 12-month-old VPS35 $5^{\mathrm{D} 620 \mathrm{~N} /+}$ knockin mice was similar to that of WT mice (Fig. 3A, B). Sixteen-month-old VPS35 ${ }^{\mathrm{D} 620 \mathrm{~N} /+}$ knockin mice displayed late-onset hypokinesia phenotype, and reduced velocity and distance of locomotor activity (Fig. 3A, B). Motor function of WT or VPS35 $5^{\mathrm{D} 620 \mathrm{~N} /+}$ mouse was also examined by conducting the pole test. $\mathrm{VPS}^{\mathrm{D} 620 \mathrm{~N} /+}$ or WT mice aged 12 months exhibited a similar time required to carry out pole test (Fig. 3C). Sixteen-month-old heterozygous VPS3 $5^{\mathrm{D} 620 \mathrm{~N} /+}$ mice displayed bradykinesia phenotype and required more time to finish pole test (Fig. 3C). L-DOPA treatment ameliorates
PD symptoms exhibited by PARK17 patients with heterozygous (D620N) VPS35 mutation $^{7,8}$. Therefore, LDOPA is expected to exert a beneficial effect on hypokinesia phenotype displayed by VPS3 $5^{\mathrm{D} 620 \mathrm{~N} /+}$ knockin mice. Intraperitoneal application of methyl L-DOPA significantly reversed reduced distance and velocity of locomotion displayed by heterozygous VPS3 $5^{\mathrm{D} 620 \mathrm{~N} /+}$ mice aged 16 months (Fig. 3D, E).

Protein level of a-synuclein or phospho-tau ${ }^{\text {Ser202/Thr205 }}$ is increased in SN of heterozygous VPS3 $5^{\mathrm{D} 620 \mathrm{~N} /+}$ knockin mice aged 16 months

Except cell death of SNpc DAergic neurons, another neuropathological finding of PD is the presence of intracellular Lewy bodies ${ }^{1}$, which contain $\alpha$-synuclein and phosphorylated $\alpha$-synuclein. Protein level of cytosolic $\alpha$ synuclein was not significantly altered in SN of 12-monthold VPS35 ${ }^{\mathrm{D} 620 \mathrm{~N} /+}$ knockin mice (Fig. 4A, B). Protein expression of cytosolic $\alpha$-synuclein was upregulated in SN of VPS35 $5^{\mathrm{D} 620 \mathrm{~N} /+}$ knockin mice at age of 16 months (Fig. 4A, B). Confocal immunofluorescence imaging study demonstrated that compared to WT mice, cytosolic level of 


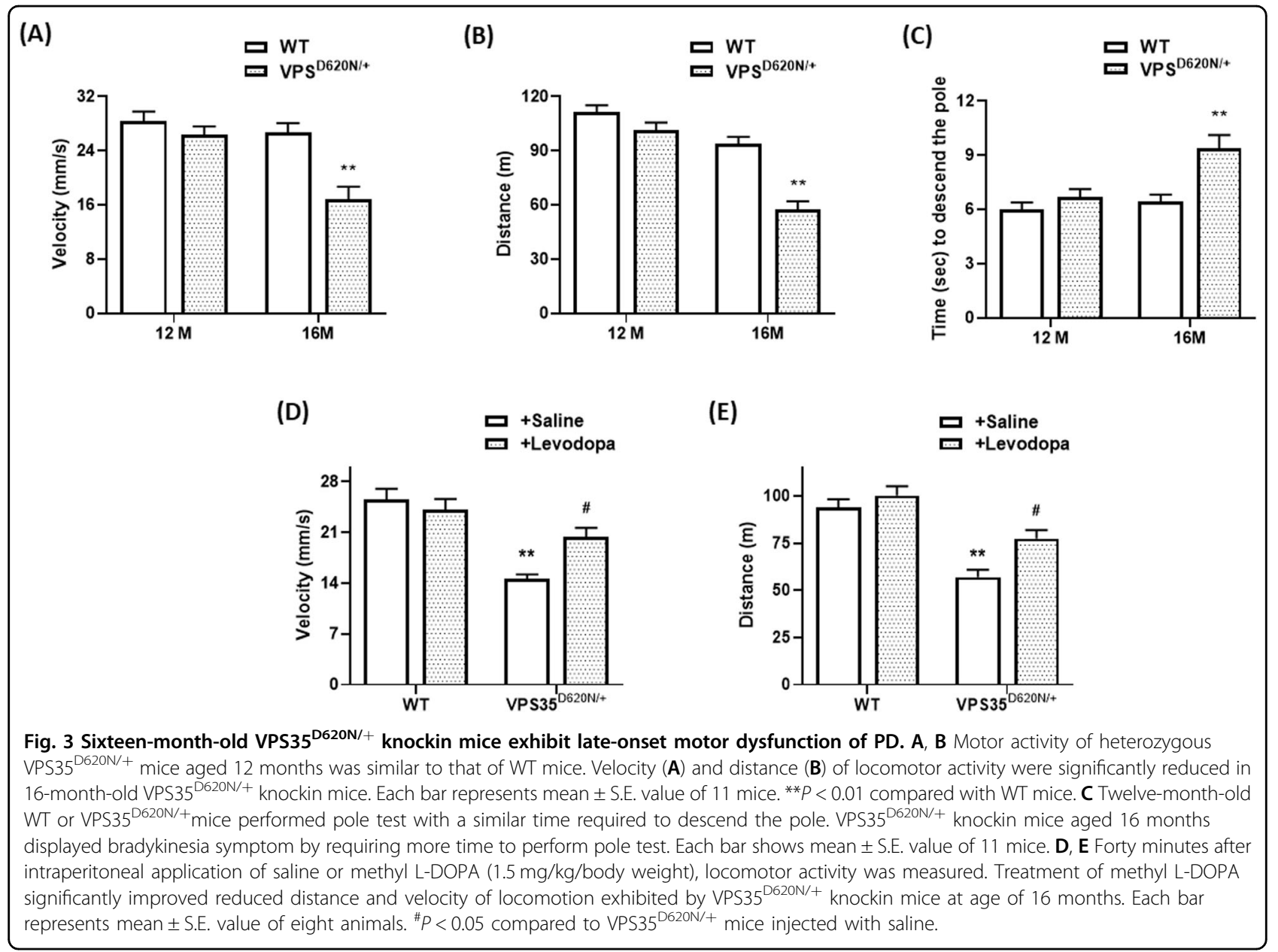

$\alpha$-synuclein was significantly increased in TH-positive SNpc DAergic neurons of VPS35 ${ }^{\mathrm{D} 620 \mathrm{~N} /+}$ mice aged 16 months (Fig. 4C). Level of cytosolic phospho- $\alpha$-synuclein ${ }^{\text {Ser129 }}$ was not altered in 12- or 16-month-old heterozygous VPS35 $5^{\text {D620N/+ }}$ mice (Fig. 4A, B). Immunocytochemical analysis using $\alpha$-synuclein antiserum or phospho- $\alpha$-synuclein $^{\text {Ser129 }}$ antibody indicated that Lewy body was absent in brain areas, including SN, hippocampus, and cerebral cortex, of 16-month-old VPS35 ${ }^{\text {D620N/+ }}$ knockin mice (data not shown; $n=5$ mice).

Tau pathology, which is believed to be caused by hyperphosphorylation of microtubule-associated protein tau, is observed in patients affected with sporadic or familial $\mathrm{PD}^{42,43}$. A similar protein expression of cytosolic tau was observed in SN of WT and VPS35 $5^{\mathrm{D} 620 \mathrm{~N} /+}$ mice aged 12 or 16 months (Fig. 4A, B). Level of cytosolic phospho-tau $^{\text {Ser202/Thr205 }}$ was upregulated in SN of 16month-old heterozygous VPS3 $5^{\mathrm{D} 620 \mathrm{~N} /+}$ mice (Fig. 4A, B). Immunohistochemical staining using anti-phosphotau ${ }^{\text {Ser202/Thr205 antibody demonstrated that tau }}$ pathology-like protein aggregates of phospho-tau were found in SN of VPS35 $5^{\mathrm{D} 620 \mathrm{~N} /+}$ knockin mice at age of 16 months ( $n=3$ mice; Fig. $4 \mathrm{D})$.

\section{Activity of Wnt/ $\beta$-catenin signaling pathway is impaired in SN of VPS35 $5^{\mathrm{D} 620 \mathrm{~N} /+}$ knockin mice aged 16 months}

In this study, it was hypothesized that mutant (D620N) VPS35 causes the dysfunction of VPS35-containing retromer complex, which results in an impaired secretion of Wnt ligand and subsequent degradation of Wnt protein. Consistent with our hypothesis, protein level of Wnt1, which activates canonical Wnt/ $\beta$-catenin pathway ${ }^{24}$, was decreased in $\mathrm{SN}$ of 16-month-old heterozygous VPS35 $5^{\text {D620N/+ }}$ mice (Fig. 5A, D).

Wnt1 activates the neuroprotective signaling cascade and promotes survival of SNpc DAergic neurons by increasing nuclear level of $\beta$-catenin ${ }^{24,30,31}$. Nuclear $\beta$-catenin is the most important downstream effector protein of $\mathrm{Wnt} / \beta$-catenin signaling cascade, and level of nuclear $\beta$-catenin reflects activity of this pathway ${ }^{24,26}$. Downregulated protein expression of Wnt1 is expected to impair activity of $\mathrm{Wnt} / \beta$-catenin signaling cascade by 
$(A)$

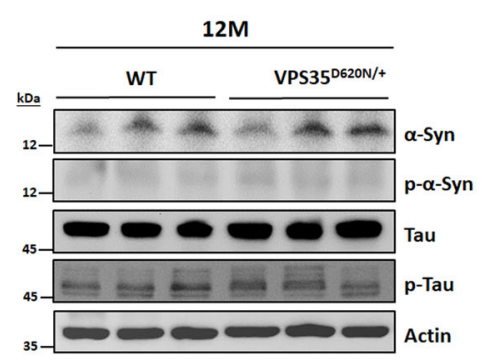

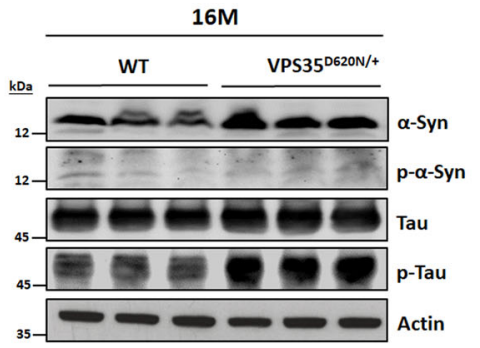

(B)

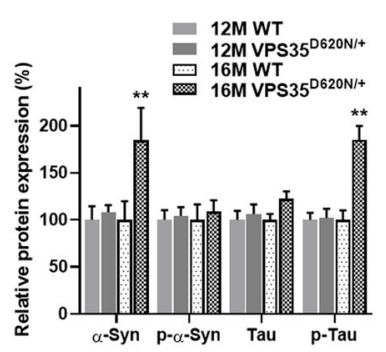

(D)
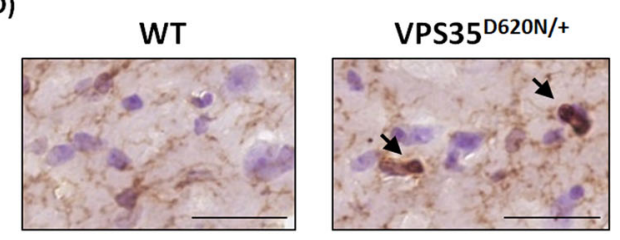

(C)

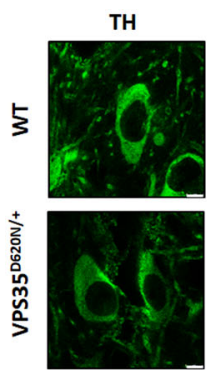

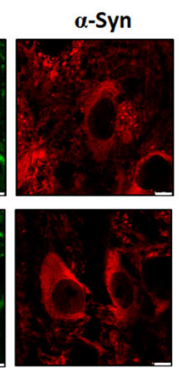
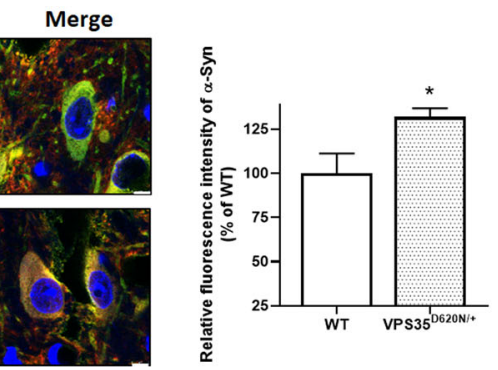

Fig. 4 Upregulated levels of $a$-synuclein and phospho-tau ${ }^{\mathrm{Ser202/Thr205}}$ were observed in SN of VPS35 ${ }^{\mathrm{D} 620 \mathrm{~N} /+}$ knockin mice at age of 16 months. A, B Protein expression of cytosolic a-synuclein (a-Syn) or phospho-tau ${ }^{\text {Ser202/Thr205 }}$ ( $p$-Tau) was upregulated in SN of 16-month-old heterozygous VPS35 $5^{\mathrm{D} 620 \mathrm{~N} /+}$ mice. Upregulated expression of a-synuclein or phospho-tau ${ }^{\mathrm{Ser} 202 / \mathrm{Th} r 205}$ was not found in SN of VPS35 age of 12 months. A similar protein level of cytosolic phospho-a-synuclein ${ }^{\text {Ser129 }}$ (p-a-Syn) or tau was found in SN of 12- or 16-month-old WT and VPS35 $5^{\mathrm{D} 620 \mathrm{~N} /+}$ mice. ${ }^{* *} P<0.01$ compared with WT mice. Each bar represents mean \pm S.E. value of seven mice. C Confocal double immunofluorescence staining showed that cytosolic expression of a-synuclein was significantly upregulated in TH-positive SNpc DAergic neurons of VPS35 ${ }^{\mathrm{D} 620 \mathrm{~N} /+}$ knockin mice at age of 16 months. ${ }^{*} P<0.05$ compared with WT mice. Each bar represents mean \pm S.E. value of 80 neurons from four mice.

D Immunocytochemical staining using anti-phospho-tau ${ }^{\text {Ser202/Thr205 }}$ antiserum showed that tau pathology-like protein aggregates of phospho-tau (arrow) were found in SN of VPS35 $5^{\mathrm{D} 20 \mathrm{~N} /+}$ mice aged 16 months. Scale bar is $30 \mu \mathrm{m}$.

decreasing protein level of nuclear $\beta$-catenin. Protein level of nuclear $\beta$-catenin was reduced in SN of 16-month-old VPS35 ${ }^{\mathrm{D} 620 \mathrm{~N} /+}$ knockin mice (Fig. 5B, D).

Nuclear $\beta$-catenin promotes the survival of neurons by upregulating expression of pro-survival and antiapoptotic protein survivin ${ }^{44,45}$, which belongs to inhibitor-of-apoptosis (IAP) family of proteins ${ }^{46}$. Mutant (D620N) VPS35-induced decrease in protein level of nuclear $\beta$-catenin is expected to downregulate protein expression of survivin. Immunoblotting assays demonstrated that level of cytosolic survivin was decreased in $\mathrm{SN}$ of heterozygous VPS35 $5^{\mathrm{D} 620 \mathrm{~N} /+}$ mice aged 16 months (Fig. 5C, D). Confocal double immunofluorescence staining showed that compared to WT mice, downregulated expression of cytosolic survivin was found in TH-positive SNpc DAergic neurons of 16-month-old VPS35 ${ }^{\mathrm{D} 620 \mathrm{~N} /+}$ knockin mice (Fig. 5E). Protein level of Wnt1, nuclear $\beta$-catenin, or survivin was not significantly altered in SN of VPS35 $5^{\mathrm{D} 620 \mathrm{~N} /+}$ mice at age of 12 months (Fig. $5 \mathrm{~A}-\mathrm{D})$.

Survivin exerts neuroprotective and antiapoptotic effect by interacting with XIAP, and inhibiting activation of caspase- 9 and caspase- 8 (ref. ${ }^{46}$ ). Downregulated expression of antiapoptotic survivin caused by mutant (D620N) VPS35 could upregulate production of active caspase- 8 and active caspase-9. Western blot study indicated that cytosolic protein level of active caspase- 9 and active caspase- 8 was upregulated in SN of VPS35 $5^{\mathrm{D} 620 \mathrm{~N} /+}$ knockin mice aged 16 months (Fig. 5C, D). Protein expression of active caspase- 8 or active caspase- 9 was not altered in SN of 12month-old VPS35 $5^{\mathrm{D} 620 \mathrm{~N} /+}$ mice (Fig. 5 C, D). Confocal immunofluorescence staining demonstrated that protein expression of active caspase- 8 or active caspase- 9 was upregulated in $\mathrm{TH}$-positive $\mathrm{SNpc}$ DAergic neurons of VPS35 $^{\text {D620N/+ }}$ mice aged 16 months (Fig. $5 \mathrm{E}$ ).

\section{Abnormal morphology of mitochondria and mitochondrial dysfunction are found in SN of 16-month-old VPS35 $5^{\text {D620N/+ }}$ knockin mice}

VPS35 participates in controlling morphology and function of mitochondria by regulating fission or fusion process of mitochondria ${ }^{10,15,39,40}$. Impaired function of VPS35 caused by $(\mathrm{D} 620 \mathrm{~N})$ mutation could lead to abnormal morphology of mitochondria in SNpc DAergic neurons. Transmission electron microscopy study demonstrated that compared to 


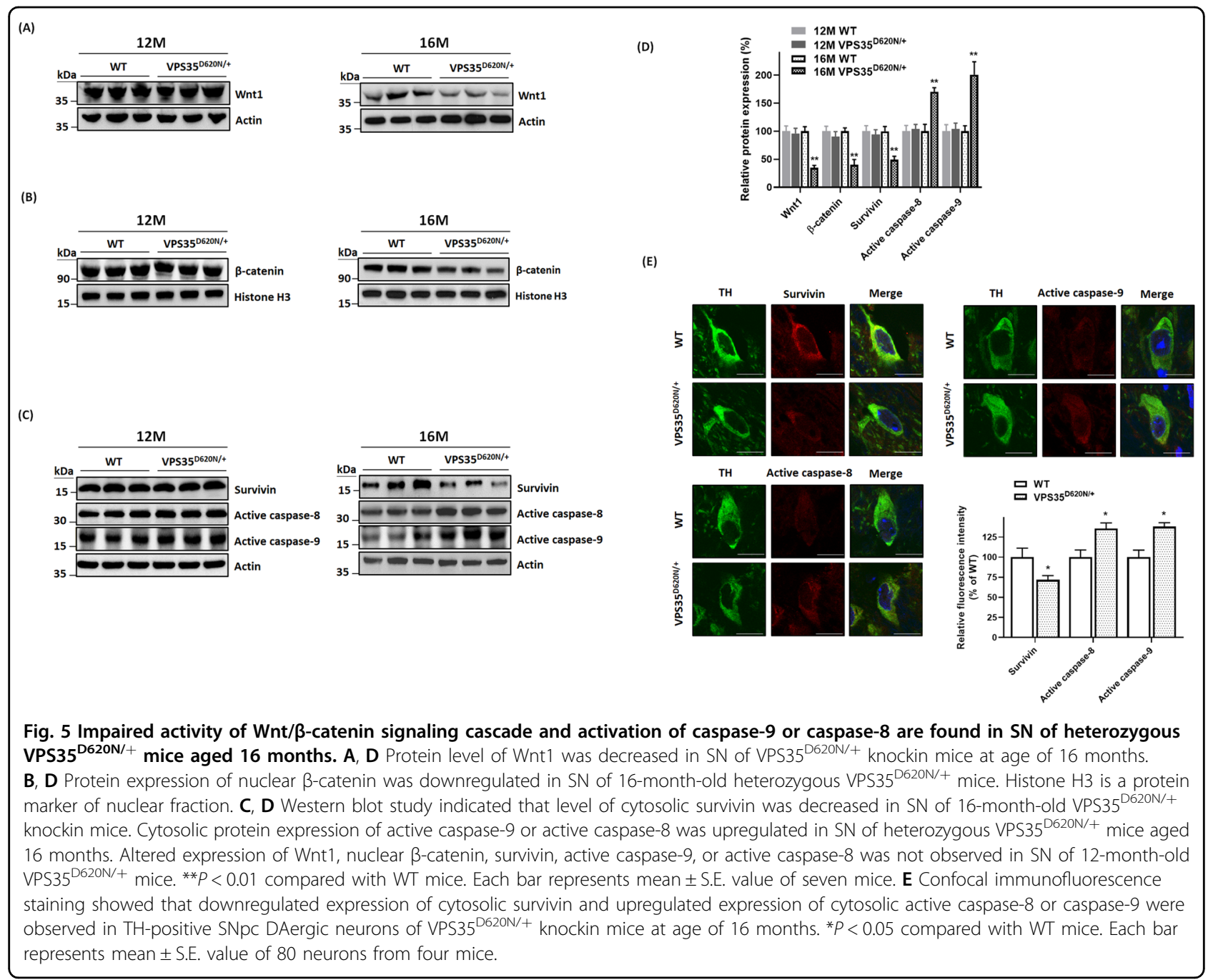

normal morphology of mitochondria found in neuromelanin organelle-containing putative SNpc DAergic cells of agematched WT mice, a significant reduction in the size of mitochondria and resulting mitochondrial fragmentation was observed in neuromelanin-positive SNpc DAergic neurons of heterozygous VPS35 $5^{\mathrm{D} 620 \mathrm{~N} /+}$ mice aged 16 months (Fig. 6A). Confocal immunofluorescence staining of Tom20, a mitochondrial marker, was conducted to visualize mitochondrial morphology of TH-positive SNpc DAergic neurons. Mitochondria displayed a normal long thread-like structure in TH-positive SNpc DAergic neurons of WT mice (Fig. 6B). Consistent with results of transmission electron microscopy study, fragmented mitochondria, which are truncated and shortened, were observed in TH-positive SNpc DAergic neurons of 16-month-old VPS35 $5^{\mathrm{D} 620 \mathrm{~N} /+}$ mice (Fig. 6B).

VPS35 deficiency has been shown to downregulate protein level of mitofusin 2 (ref. ${ }^{39}$ ), which is required for mitochondrial fusion. An in vitro study reported that mutant (D620N) VPS35 caused dysregulated expression of dynamin-related protein 1 (Drp1 $)^{40}$, which is needed for mitochondrial fission. Protein expression of mitochondrial mitofusin 2 was not altered in $\mathrm{SN}$ of VPS35 $5^{\text {D620N/+ }}$ knockin mice at age of 12 months (Fig. 6C). Protein level of mitochondrial mitofusin 2 was decreased in SN of 16-month-old VPS35 ${ }^{\mathrm{D} 620 \mathrm{~N} /+}$ knockin mice (Fig. 6C). A similar expression of mitochondrial Drp1 was found in SN of WT or VPS35 $5^{\mathrm{D} 620 \mathrm{~N} /+}$ mice aged 12 or 16 months (Fig. 6C). Confocal immunofluorescence staining showed that compared to WT mice, protein expression of mitofusin 2 was significantly downregulated in TH-positive SNpc DAergic neurons of 16-month-old VPS35 ${ }^{\text {D620N/+ }}$ knockin mice (Fig. 6D).

Mutant (D620N) VPS35-induced abnormal morphology of mitochondria could cause the malfunction of mitochondria and impair activity of mitochondrial complex I-IV. Mitochondrial complex I and IV activities were reduced in SN of 16-month-old heterozygous VPS35 $5^{\mathrm{D} 620 \mathrm{~N} /+}$ mice (Fig. 6E). A similar activity of mitochondrial complex II or complex III was observed in SN of WT or VPS35 $5^{\mathrm{D} 620 \mathrm{~N} /+}$ mice aged 16 months (Fig. 6E). 


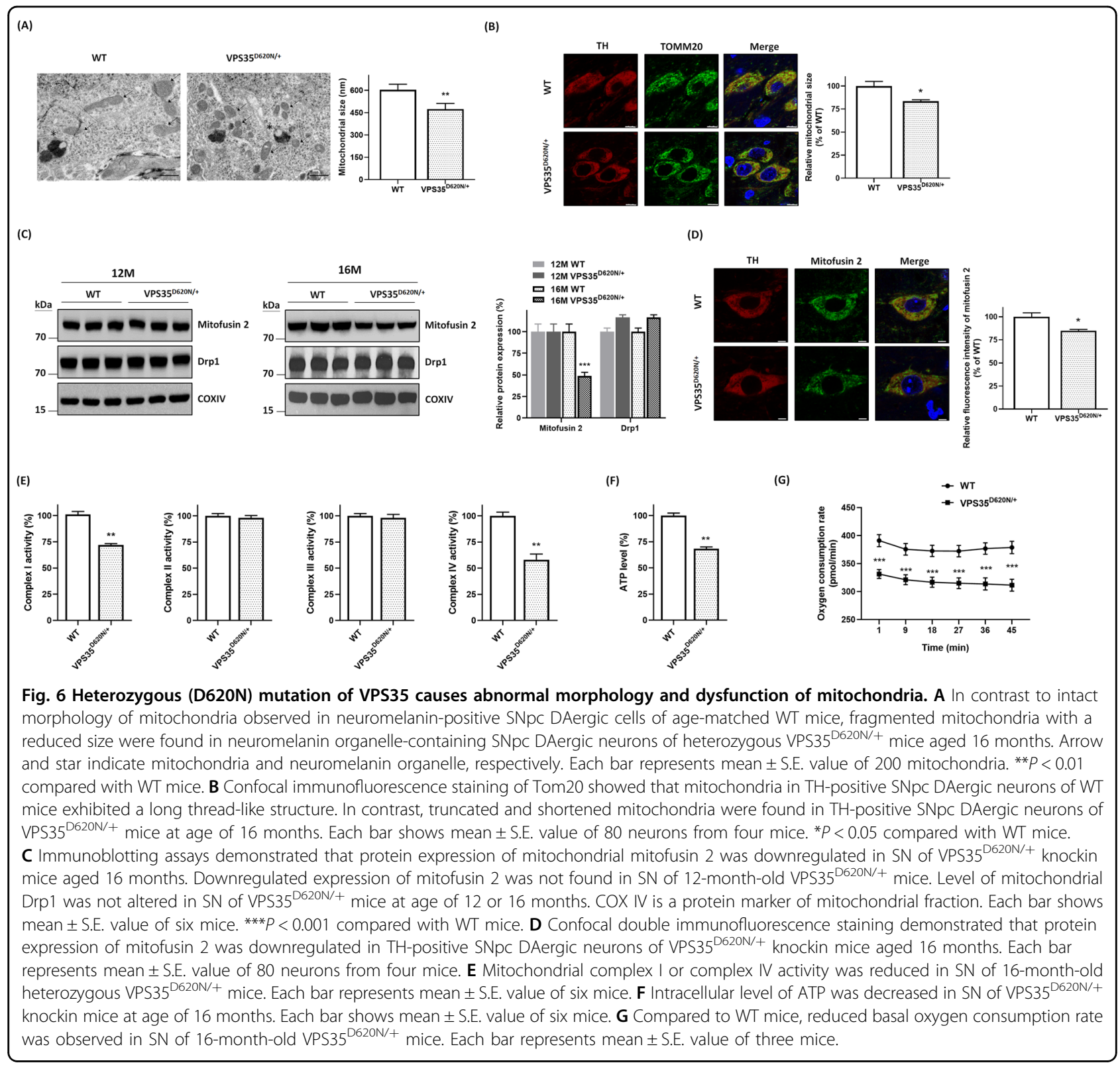

As a result of mitochondrial dysfunction, a significant reduction of intracellular ATP level was observed in SN of 16-month-old VPS35 $5^{\mathrm{D} 620 \mathrm{~N} /+}$ knockin mice (Fig. 6F). Mutant (D620N) VPS35-induced mitochondrial dysfunction also led to the reduced basal oxygen consumption rate and impaired mitochondrial respiration in $\mathrm{SN}$ of VPS35 $5^{\text {D620N/+ }}$ mice aged 16 months (Fig. 6G).

\section{Overgeneration of mitochondrial ROS, oxidative stress,} and activation of mitochondrial apoptotic cascade are observed in SN of heterozygous VPS3 $5^{\mathrm{D} 620 \mathrm{~N} /+}$ mice aged 16 months

Mutant (D620N) VPS35-induced impairment of mitochondrial function is expected to enhance generation of mitochondrial ROS and lipid peroxidation of mitochondria, which results in causing oxidative stress. An upregulated formation of mitochondrial ROS was found in SN of VPS35 $5^{\mathrm{D} 620 \mathrm{~N} /+}$ knockin mice at age of 16 months (Fig. 7A). TBARS assay was performed to evaluate lipid peroxidation of mitochondria. A pronounced increase in mitochondrial lipid peroxidation was observed in $\mathrm{SN}$ of 16-month-old heterozygous VPS35 $5^{\mathrm{D} 620 \mathrm{~N} /+}$ mice (Fig. 7B).

Overgeneration of mitochondrial ROS-induced oxidative stress is believed to cause cytochrome c release from mitochondria to cytosol and activation of mitochondria-mediated apoptotic pathway ${ }^{36,37}$. Confocal immunofluorescence staining showed that protein level of cytosolic cytochrome c was significantly 
(A)

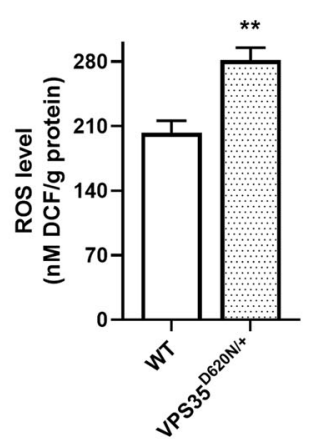

(B)

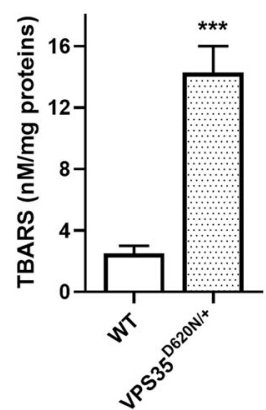

(C)
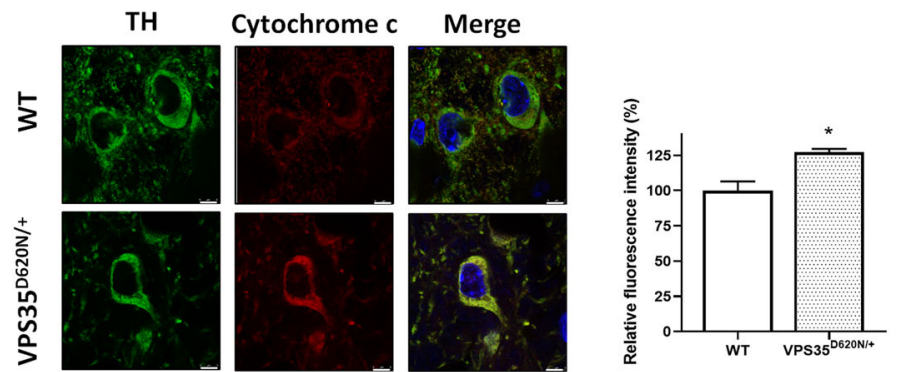

(D)

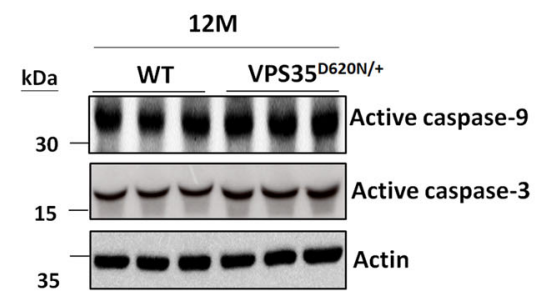

(E)

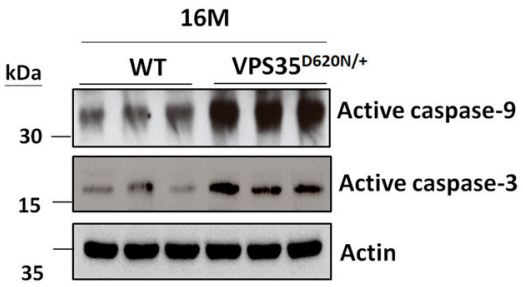

(F)

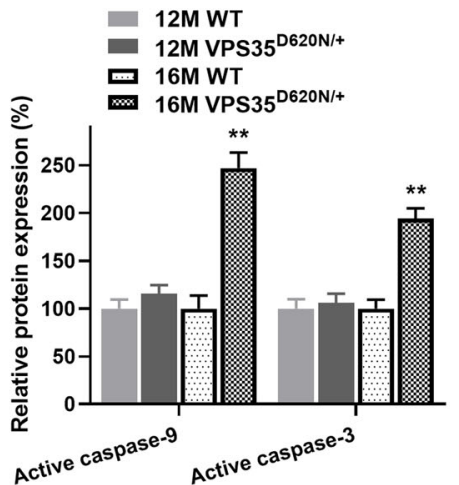

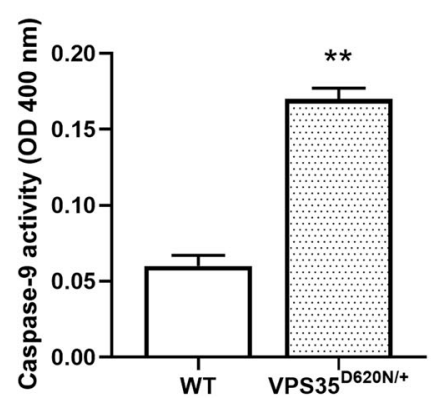

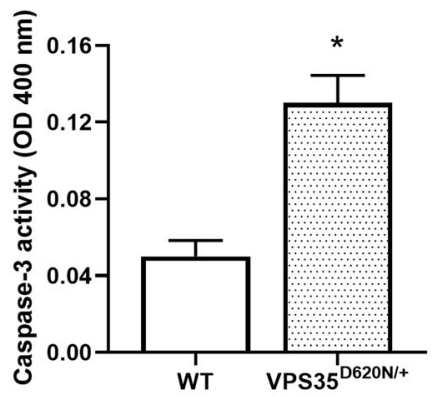

Fig. 7 Heterozygous (D620N) mutation of VPS35 causes overgeneration of mitochondrial ROS, oxidative stress, and activation of mitochondria-mediated apoptotic pathway. A Level of mitochondrial ROS was upregulated in SN of heterozygous VPS35 ${ }^{\mathrm{D} 620 \mathrm{~N} /+}$ mice aged 16 months. Each bar represents mean \pm S.E. value of six mice. ${ }^{* *} P<0.01$ compared with WT mice. B Compared to WT mice, lipid peroxidation of mitochondria was markedly enhanced in SN of 16-month-old VPS35 ${ }^{\mathrm{D} 620 \mathrm{~N} /+}$ knockin mice. Each bar shows mean \pm S.E. value of six mice. ${ }^{* * *} P<0.001$ compared to WT mice. C Confocal double immunofluorescence staining demonstrated that protein expression of cytosolic cytochrome c was significantly upregulated in SNpc DAergic neurons of VPS35 ${ }^{\mathrm{D} 620 \mathrm{~N} /+}$ mice aged 16 months. Each bar represents mean \pm S.E. value of 80 neurons from four mice. ${ }^{*} P<0.05$ compared with WT mice. D Cytosolic protein levels of active caspase-9 and active caspase-3 were increased in SN of heterozygous VPS35 ${ }^{\mathrm{D} 620 \mathrm{~N} /+}$ mice aged 16 months. Each bar represents mean \pm S.E. value of seven mice. $\mathbf{E}$ and $\mathbf{F}$ The activity of caspase-9 or caspase3 was significantly increased in SN of VPS35 $5^{\text {D620N/+ }}$ knockin mice at age of 16 months. Each bar shows mean \pm S.E. value of four mice.

increased in TH-positive SNpc DAergic neurons of heterozygous VPS35 ${ }^{\mathrm{D} 620 \mathrm{~N} /+}$ mice aged 16 months (Fig. $7 C)$. Protein levels of cytosolic active caspase- 9 and active caspase-3 were increased in SN of 16-month-old VPS35 $5^{\text {D620N/+ }}$ knockin mice (Fig. 7D). Protein expression of active caspase- 9 or active caspase- 3 was not significantly altered in SN of VPS35 $5^{\mathrm{D} 62 \mathrm{~N} /+}$ mice aged 12 months (Fig. 7D). Consistent with results of western blot analysis, activity of caspase-9 (Fig. 7E) or caspase-3 (Fig. 7F) was significantly upregulated in $\mathrm{SN}$ of VPS3 $5^{\text {D620N/+ }}$ knockin mice at age of 16 months.

\section{Discussion}

Patients with PARK17 display autosomal dominant inheritance $^{3-8}$. Heterozygous (D620N) mutation of VPS35 has been confirmed as the genetic cause of PARK17 
(refs. ${ }^{7,8}$ ). To better understand molecular pathogenic pathways by which mutant (D620N) VPS35 causes neurodegeneration of SNpc DAergic cells and resulting PD symptoms, we generated heterozygous VPS35 $5^{\text {D620N/+ }}$ knockin mouse, which is an ideal animal model of (D620N) VPS35-induced autosomal dominant PARK17. In accordance with previous studies indicating that heterozygous (D620N) mutation of VPS35 causes late-onset PARK17 (refs. ${ }^{3,5,7,8}$ ), heterozygous VPS35 $5^{\mathrm{D} 620 \mathrm{~N} /+}$ knockin mice aged 16 months exhibited late-onset loss of SNpc DAergic neurons and decrease in density of $\mathrm{TH}$-positive nigrostriatal DAergic terminals. As a result, 16-month-old VPS35 ${ }^{\mathrm{D} 620 \mathrm{~N} /+}$ knockin mice displayed late-onset hypokinesia, which was indicated by a decrease in locomotor velocity or locomotive distance, and bradykinesia, which was demonstrated by a longer time needed to carry out pole test. Consistent with clinical studies demonstrating that L-DOPA treatment exerts a beneficial effect on PARK17 patients carrying (D620N) VPS35 mutation ${ }^{7,8}$, intraperitoneal injection of methyl L-DOPA significantly ameliorated hypokinesia phenotype displayed by heterozygous VPS3 $5^{\mathrm{D} 620 \mathrm{~N} /+}$ mice at age of 16 months.

In accordance with our finding, late-onset neurodegeneration of SNpc DAergic cells was also observed in (D620N) VPS35 knockin mice prepared by another group ${ }^{47}$. The loss of DAergic axon terminals in the ST of VPS35 ${ }^{\text {D620N/+ }}$ knockin mice, which is indicated by a reduction in the density of striatal TH staining, is expected to result in an impairment of evoked dopamine release from nigrostriatal DAergic terminals. Consistent with this hypothesis, previous in vivo microdialysis study reported that evoked dopamine release was significantly impaired in the caudate putamen of 5-7-month-old (D620N) VPS35 knockin mice ${ }^{48}$. On the other hand, an augmented dopamine release from striatal slices was observed in 3month-old (D620N) VPS35 knockin mice ${ }^{49}$.

Up to now, postmortem neuropathological examination has not been conducted to visualize the formation of Lewy bodies, which contain $\alpha$-synuclein and phosphorylated $\alpha$ synuclein, in the brain of PARK17 patients with (D620N) VPS35 mutation. Our immunocytochemical staining study indicated that Lewy bodies were not found in brain regions of VPS35 $5^{\mathrm{D} 620 \mathrm{~N} /+}$ knockin mice aged 16 months. Interestingly, upregulated level of cytosolic $\alpha$-synuclein was observed in SN of VPS35 $5^{\mathrm{D} 620 \mathrm{~N} /+}$ mice aged 16 months. One of important pathways that participate in the degradation of $\alpha$-synuclein is chaperone-mediated autophagy $^{50-52}$. Lamp2a is the lysosomal membrane protein for CMA and required for CMA-mediated degradation of $\alpha$-synuclein. VPS35, a critical component of retromer multi-subunit complex ${ }^{14-17}$, is believed to mediate endosome-to-TGN retrieval of Lamp2a and maintain normal Lamp2a level ${ }^{52}$. (D620N) VPS35 mutation impairs cargo sorting function of retromer and causes trafficking defects ${ }^{34}$. It is very likely that defective trafficking and reduced protein level of Lamp2a caused by mutant (D620N) VPS35 results in impairment of CMAmediated degradation of $\alpha$-synuclein, and increased level of $\alpha$-synuclein in $\mathrm{SN}$ of 16-month-old heterozygous VPS35 $5^{\mathrm{D} 620 \mathrm{~N} /+}$ mice.

Accumulation of hyperphosphorylated microtubuleassociated protein tau could cause tau pathology, which is observed in patients affected with sporadic or hereditary $\mathrm{PD}^{42,43}$. Interestingly, increased protein level of phospho$\mathrm{tau}^{\mathrm{Ser202/Thr205}}$ was found in SN of VPS35 $5^{\mathrm{D} 620 \mathrm{~N} /+}$ mice aged 16 months. Tau pathology-like protein aggregates of phospho-tau ${ }^{\mathrm{Ser} 202 / \mathrm{Thr} 205}$ were observed in SN of 16-monthold VPS $35^{\mathrm{D} 620 \mathrm{~N} /+}$ knockin mice. Future study is needed to clarify molecular mechanism underlying mutant (D620N) VPS35-induced phosphorylation of tau. Consistent with our result, tau pathology was also found in the brain of (D620N) VPS35 knockin mice prepared by others ${ }^{47}$. Hyperphosphorylated tau is believed to depolymerize microtubules and cause neuronal dysfunction ${ }^{42}$. The possible involvement of upregulated phospho-tau ${ }^{\mathrm{Ser202/Th205}}$ level in mutant (D620N) VPS35-induced degeneration of SNpc DAergic neurons remains to be studied.

Several lines of evidence indicate that Wnt protein is involved in promoting development and survival of SNpc DAergic neurons through activating $W n t / \beta$-catenin pathway $^{27-33}$. Wnt1 activates canonical Wnt/ $\beta$-catenin signaling pathway, and facilitates differentiation and survival of SN DAergic neurons ${ }^{30,31}$. Normal expression of $\beta$-catenin is required for survival and maintenance of SNpc DAergic neurons $^{33}$. Autocrine or paracrine secretion of Wnt ligands is needed for normal activity of Wnt-mediated pathways. VPS35-containing retromer complex plays a vital role in normal Wnt secretion by recycling Wntless, which mediates release of Wnt, from endosomes to $\mathrm{TGN}^{19-23}$. Therefore, normal function of VPS35 is required for Wnt secretion and $\mathrm{Wnt} / \beta$-catenin signaling activity. In this study, we hypothesized that (D620N) VPS35 mutation causes malfunction of VPS35-containing retromer, which results in a defective secretion of Wnt ligand and subsequent degradation of Wnt protein. In accordance with this hypothesis, level of Wnt1 protein was downregulated in $\mathrm{SN}$ of heterozygous VPS35 $5^{\mathrm{D} 620 \mathrm{~N} /+}$ mice aged 16 months. Wnt1 promotes survival of SNpc DAergic neurons by increasing protein level of nuclear $\beta$-catenin ${ }^{24,30,31}$, which indicates activity of Wnt/ $\beta$-catenin cascade ${ }^{24,26}$. Downregulated level of Wnt1 protein led to the impairment of $\mathrm{Wnt} / \beta$-catenin pathway by significantly decreasing nuclear $\beta$-catenin in $\mathrm{SN}$ of VPS35 $5^{\text {D620N/+ }}$ knockin mice at age of 16 months. Nuclear $\beta$-catenin exerts a neuroprotective effect by increasing expression of pro-survival and antiapoptotic protein sur$\operatorname{vivin}^{44,45}$. Downregulated expression of nuclear $\beta$-catenin caused a significant decrease of cytosolic survivin in $\mathrm{SN}$ of 16-month-old VPS35 $5^{\mathrm{D} 620 \mathrm{~N} /+}$ mice. Survivin belongs to IAP family of proteins and induces an antiapoptotic and 
neuroprotective effect by inhibiting activation of caspase- 9 and caspase- $8^{46}$. Decreased protein level of cytosolic survivin resulted in upregulated levels of active caspase- 9 and active caspase- 8 in SN of heterozygous VPS $35^{\mathrm{D} 620 \mathrm{~N} /+}$ mice at age of 16 months. Our results suggest that mutant (D620N) VPS35 causes degeneration of SNpc DAergic neurons and resulting PARK17 by impairing activity of Wnt/ $\beta$-catenin neuroprotective signaling cascade. Consistent with our finding, dysregulated Wnt $/ \beta$-catenin signaling has also been implicated in pathogenesis of sporadic $\mathrm{PD}^{53}$.

Dynamic process of mitochondrial fusion and fission regulates normal morphology and function of mitochondria $^{36,37,54}$. VPS35 is involved in controlling mitochondrial dynamics and morphology by regulating process of mitochondrial fission or fusion ${ }^{10,15,39,40,55}$. PARK17 (D620N) mutation-induced dysfunction of VPS35 could result in abnormal morphology of mitochondria in SNpc DAergic neurons. Consistent with this hypothesis, a significant decrease in mitochondrial size and resulting mitochondrial fragmentation was found in SNpc DAergic neurons of 16month-old VPS35 $5^{\mathrm{D} 620 \mathrm{~N} /+}$ knockin mice. A previous study reported that VPS35 deficiency could cause mitochondrial fragmentation in DAergic neurons by decreasing protein level of mitofusin 2 and impairing mitochondrial fusion ${ }^{39}$. In vitro studies suggested that mutant (D620N) VPS35 interacts with Drp1 and causes dysregulated expression of Drp1, which leads to augmented fission process and mitochondrial fragmentation ${ }^{40,55}$. Therefore, mitochondrial fragmentation observed in SNpc DAergic neurons of VPS35 ${ }^{\mathrm{D} 62 \mathrm{~N} /+}$ mice could result from downregulated expression of mitofusin 2 or upregulated expression of Drp1. Immunoblotting assay showed that protein level of mitochondrial mitofusin 2 was decreased in $\mathrm{SN}$ of heterozygous VPS35 $5^{\mathrm{D} 620 \mathrm{~N} /+}$ mice at age of 16 months and that Drp1level was not altered in SN of VPS35 $5^{\mathrm{D} 620 \mathrm{~N} /+}$ mice. Interestingly, downregulated protein expression of mitofusin 2 was also observed in frontal cortex and hippocampus of Alzheimer's disease patients ${ }^{56}$.

Mutant (D620N) VPS35-induced mitochondrial fragmentation is expected to cause dysfunction of mitochondria. As a result, impaired mitochondrial complex I or complex IV activity, reduced intracellular ATP level and impaired mitochondrial respiration were observed in $\mathrm{SN}$ of heterozygous $\mathrm{VPS}^{\mathrm{D} 620 \mathrm{~N} /+}$ mice aged 16 months. Consistent with our finding, mitochondrial complex I activity was reduced in fibroblasts obtained from a PARK17 patient with (D620N) VPS35 mutation ${ }^{57}$. Impairment of mitochondrial function and complex I activity led to the mitochondrial ROS overgeneration and oxidative stress, which was indicated by increased mitochondrial ROS level and lipid peroxidation in SN of 16-month-old VPS35 $5^{\mathrm{D} 620 \mathrm{~N} /+}$ knockin mice. Upregulated generation of mitochondrial ROS and oxidative stress caused activation of mitochondria-mediated apoptotic cascade by increasing cytosolic level of cytochrome c, active caspase-9 or active caspase-3 in SN of 16-month-old heterozygous VPS35 $5^{\mathrm{D} 620 \mathrm{~N} /+}$ mice. Our findings suggest that PARK17 mutant (D620N) VPS35 causes neurodegeneration of SNpc DAergic cells by causing mitochondrial fragmentation and dysfunction of mitochondria, which leads to overproduction of mitochondrial ROS and activation of mitochondrial apoptotic pathway.

In summary, we prepared animal model of autosomal dominant and late-onset PARK17 by generating heterozygous VPS35 ${ }^{\mathrm{D} 620 \mathrm{~N} /+}$ knockin mice, which exhibit late-onset neurodegeneration of SNpc DAergic cells and motor dysfunction phenotypes of parkinsonism. The results of our study suggest that PARK17 mutant (D620N) VPS35 induces degeneration of SNpc DAergic neurons by impairing the activity of $\mathrm{Wnt} / \mathrm{\beta}$-catenin signaling cascade, and causing abnormal morphology and malfunction of mitochondria.

\section{Acknowledgements}

This study was supported by the Ministry of Science and Technology, Taiwan (MOST106-2314-B-182A-012-MY3 and MOST109-2314-B-182A-072-MY3 to C.-C. C.; MOST 103-2314-B-182A-119-MY3 to Y.-H.W.; MOST 104-2320-B-182-014-MY3 and MOST107-2320-B-182-036-MY3 to H.-L.W.), Chang Gung Memorial Hospital, Taiwan (CMRPG3K0951-2 and CMRPG3J0761-3 to C.-C.C.; CMRPG3D1293 to Y.-H.W.; CMRPD1C0623, CRRPD1C0013, CMRPD180433, CMRPD1B0332, EMRPD1G0171, and CMRPD1H0282 to H.-L.W.), and Healthy Aging Research Center (EMRPD1H0551, EMRPD1H0381, EMRPD1K0481, and EMRPD1K0361), Chang Gung University, Taiwan. The authors thank technical assistance of Clinical Proteomics Core Laboratory and Microscopy Core Laboratory, Chang Gung Memorial Hospital at Linkou.

\section{Author details \\ ${ }^{1}$ Neuroscience Research Center, Chang Gung Memorial Hospital at Linkou, Taoyuan, Taiwan. ${ }^{2}$ Department of Nursing, Chang Gung University of Science and Technology, Taoyuan, Taiwan. ${ }^{3}$ Department of Medical Biotechnology and Laboratory Science, Chang Gung University College of Medicine, Taoyuan, Taiwan. ${ }^{4}$ Healthy Aging Research Center, Chang Gung University College of Medicine, Taoyuan, Taiwan. ${ }^{5}$ Division of Movement Disorders, Department of Neurology, Chang Gung Memorial Hospital at Linkou, Taoyuan, Taiwan. \\ ${ }^{6}$ College of Medicine, Chang Gung University, Taoyuan, Taiwan. Institute of Cognitive Neuroscience, National Central University, Taoyuan, Taiwan. ${ }^{8}$ Division of Sports Medicine, Landseed International Hospital, Taoyuan, Taiwan. ${ }^{9}$ Department of Neurology, Taipei Medical University Hospital, Taipei, Taiwan. ${ }^{10}$ Department of Physiology and Pharmacology, Chang Gung University College of Medicine, Taoyuan, Taiwan}

Conflict of interest

The authors declare that they have no conflict of interest.

\section{Publisher's note}

Springer Nature remains neutral with regard to jurisdictional claims in published maps and institutional affiliations.

Supplementary Information accompanies this paper at (https://doi.org/ 10.1038/s41419-020-03228-9).

Received: 20 April 2020 Revised: 11 November 2020 Accepted: 12 November 2020

Published online: 30 November 2020

\footnotetext{
References

1. Balestrino, R. \& Schapira, A. H. V. Parkinson disease. Eur. J. Neurol. 27, 27-42 (2020).
} 
2. Moore, D. J., West, A. B., Dawson, V. L. \& Dawson, T. M. Molecular pathophysiology of Parkinson's disease. Annu. Rev. Neurosci. 28, 57-87 (2005).

3. Blauwendraat, C., Nalls, M. A. \& Singleton, A. B. The genetic architecture of Parkinson's disease. Lancet Neurol. 19, 170-178 (2020).

4. Sardi, S. P., Cedarbaum, J. M. \& Brundin, P. Targeted therapies for Parkinson's disease: from genetics to the clinic. Mov. Disord. 33, 684-696 (2018).

5. Hernandez, D. G., Reed, X. \& Singleton, A. B. Genetics in Parkinson disease: Mendelian versus non-Mendelian inheritance. J. Neurochem. 139, 59-74 (2016).

6. Bonifati, V. Genetics of Parkinson's disease-state of the art, 2013. Parkinsonism Relat. Disord. 20, S23-S28 (2014).

7. Zimprich, A. et al. A mutation in VPS35, encoding a subunit of the retromer complex, causes late-onset Parkinson disease. Am. J. Hum. Genet. 89, 168-175 (2011).

8. Vilarino-Guell, C. et al. VPS35 mutations in Parkinson disease. Am. J. Hum. Genet. 89, 162-167 (2011).

9. Mohan, M. \& Mellick, G. D. Role of the VPS35 D620N mutation in Parkinson's disease. Parkinsonism Relat. Disord. 36, 10-18 (2017).

10. Rahman, A. A. \& Morrison, B. E. Contributions of VPS35 mutations to Parkinson's disease. Neuroscience 401, 1-10 (2019).

11. Sharma, M. et al. A multi-centre clinico-genetic analysis of the VPS35 gene in Parkinson disease indicates reduced penetrance for disease-associated variants. J. Med. Genet. 49, 721-726 (2012).

12. Doyle, A., McGarry, M. P., Lee, N. A. \& Lee, J. J. The construction of transgenic and gene knockout/knockin mouse models of human disease. Transgenic Res. 21, 327-349 (2012).

13. Tsika, E. et al. Parkinson's disease-linked mutations in VPS35 induce dopaminergic neurodegeneration. Hum. Mol. Genet. 23, 4621-4638 (2014).

14. Seaman, M. N. The retromer complex - endosomal protein recycling and beyond. J. Cell Sci. 125, 4693-4702 (2012)

15. Williams, E. T., Chen, X. \& Moore, D. J. VPS35, the retromer complex and Parkinson's disease. J. Parkinsons Dis. 7, 219-233 (2017)

16. Cui, Y., Yang, Z. \& Teasdale, R. D. The functional roles of retromer in Parkinson's disease. FEBS Lett. 592, 1096-1112 (2018).

17. Abubakar, Y. S., Zheng, W., Olsson, S. \& Zhou, J. Updated Insight into the physiological and pathological roles of the retromer complex. Int. J. Mol. Sci. 18, 1601 (2017).

18. Bonifacino, J. S. \& Hurley, J. H. Retromer. Curr. Opin. Cell Biol. 20, 427-436 (2008).

19. Herr, P., Hausmann, G. \& Basler, K. WNT secretion and signalling in human disease. Trends Mol. Med. 18, 483-493 (2012).

20. Banziger, $\mathrm{C}$. et al. Wntless, a conserved membrane protein dedicated to the secretion of Wnt proteins from signaling cells. Cell 125, 509-522 (2006).

21. Belenkaya, T. Y. et al. The retromer complex influences Wnt secretion by recycling wntless from endosomes to the trans-Golgi network. Dev. Cell 14, 120-131 (2008).

22. Harterink, M. et al. A SNX3-dependent retromer pathway mediates retrograde transport of the Wnt sorting receptor Wntless and is required for Wnt secretion. Nat. Cell Biol. 13, 914-923 (2011).

23. Zhang, P., Wu, Y., Belenkaya, T. Y. \& Lin, X. SNX3 controls Wingless/Wnt secretion through regulating retromer-dependent recycling of Wntless. Cell Res. 21, 1677-1690 (2011).

24. Niehrs, C. The complex world of WNT receptor signalling. Nat. Rev. Mol. Cell Biol. 13, 767-779 (2012).

25. Berwick, D. C. \& Harvey, K. The regulation and deregulation of Wnt signaling by PARK genes in health and disease. J. Mol. Cell Biol. 6, 3-12 (2014).

26. Kahn, M. Can we safely target the WNT pathway? Nat. Rev. Drug Discov. 13, 513-532 (2014)

27. Arenas, E. Wnt signaling in midbrain dopaminergic neuron development and regenerative medicine for Parkinson's disease. J. Mol. Cell Biol. 6, 42-53 (2014).

28. Marchetti, B. Wnt/beta-catenin signaling pathway governs a full program for dopaminergic neuron survival, neurorescue and regeneration in the MPTP mouse model of Parkinson's disease. Int. J. Mol. Sci. 19, 3743-3760 (2018).

29. L'Episcopo, F. et al. A Wnt1 regulated Frizzled-1/beta-Catenin signaling pathway as a candidate regulatory circuit controlling mesencephalic dopaminergic neuron-astrocyte crosstalk: therapeutical relevance for neuron survival and neuroprotection. Mol. Neurodegener. 6, 49 (2011).

30. Castelo-Branco, G. et al. Differential regulation of midbrain dopaminergic neuron development by Wnt-1, Wnt-3a, and Wnt-5a. Proc. Natl Acad. Sci. USA 100, 12747-12752 (2003).
31. Yang, J. et al. Dynamic temporal requirement of Wnt1 in midbrain dopamine neuron development. Development 140, 1342-1352 (2013).

32. Wei, L. et al. Activation of Wnt/beta-catenin pathway by exogenous Wnt1 protects SH-SY5Y cells against 6-hydroxydopamine toxicity. J. Mol. Neurosci. 49, 105-115 (2013).

33. Dai, T. L. et al. Depletion of canonical Wnt signaling components has a neuroprotective effect on midbrain dopaminergic neurons in an MPTP-induced mouse model of Parkinson's disease. Exp. Ther. Med. 8, 384-390 (2014).

34. Follett, J. et al. The Vps35 D620N mutation linked to Parkinson's disease disrupts the cargo sorting function of retromer. Traffic 15, 230-244 (2014).

35. Deng, H., Gao, K. \& Jankovic, J. The VPS35 gene and Parkinson's disease. Mov. Disord. 28, 569-575 (2013).

36. Luo, Y., Hoffer, A., Hoffer, B. \& Qi, X. Mitochondria: a therapeutic target for Parkinson's disease? Int. J. Mol. Sci. 16, 20704-20730 (2015).

37. Bose, A. \& Beal, M. F. Mitochondrial dysfunction in Parkinson's disease. J. Neurochem. 139, 216-231 (2016).

38. Grunewald, A., Kumar, K. R. \& Sue, C. M. New insights into the complex role of mitochondria in Parkinson's disease. Prog. Neurobiol. 177, 73-93 (2019).

39. Tang, F. L. et al. VPS35 deficiency or mutation causes dopaminergic neuronal loss by impairing mitochondrial fusion and function. Cell Rep. 12, 1631-1643 (2015).

40. Wang, W. et al. Parkinson's disease-associated mutant VPS35 causes mitochondrial dysfunction by recycling DLP1 complexes. Nat. Med. 22, 54-63 (2016).

41. Chiu, C. C. et al. PARK14 (D331Y) PLA2G6 causes early-onset degeneration of substantia nigra dopaminergic neurons by inducing mitochondrial dysfunction, ER stress, mitophagy impairment and transcriptional dysregulation in a knockin mouse model. Mol. Neurobiol. 56, 3835-3853 (2019).

42. Zhang, X. et al. Tau pathology in Parkinson's disease. Front. Neurol. 9, 809 (2018).

43. Feng, S. T. et al. Update on the association between alpha-synuclein and tau with mitochondrial dysfunction: Implications for Parkinson's disease. Eur. J. Neurosci. https://doi.org/10.1111/ejn.14699 (2020). Online ahead of print.

44. Jiang, J. et al. Downregulation of the Wnt/beta-catenin signaling pathway is involved in manganese-induced neurotoxicity in rat striatum and PC12 cells. J. Neurosci. Res. 92, 783-794 (2014).

45. Zhan, L. et al. Hypoxic postconditioning activates the Wnt/beta-catenin pathway and protects against transient global cerebral ischemia through Dkk1 Inhibition and GSK-3beta inactivation. FASEB J. 33, 9291-9307 (2019).

46. Wheatley, S. P. \& Altieri, D. C. Survivin at a glance. J. Cell Sci. 132, jcs223826 (2019).

47. Chen, X. et al. Parkinson's disease-linked D620N VPS35 knockin mice manifest tau neuropathology and dopaminergic neurodegeneration. Proc. Natl Acad. Sci. USA 116, 5765-5774 (2019).

48. Ishizu, $\mathrm{N}$. et al. Impaired striatal dopamine release in homozygous vps35 D620N knock-in mice. Hum. Mol. Genet. 25, 4507-4517 (2016).

49. Cataldi, S. et al. Altered dopamine release and monoamine transporters in Vps35 p.D620N knock-in mice. NPJ Parkinsons Dis. 4, 27 (2018).

50. Xilouri, M., Brekk, O. R. \& Stefanis, L. Autophagy and Alpha-Synuclein: Relevance to Parkinson's Disease and Related Synucleopathies. Mov. Disord 31, 178-192 (2016).

51. Eleuteri, S. \& Albanese, A. VPS35-based approach: a potential innovative treatment in Parkinson's disease. Front. Neurol. 10, 1272 (2019).

52. Tang, F. L. et al. VPS35 in dopamine neurons is required for endosome-togolgi retrieval of Lamp2a, a receptor of chaperone-mediated autophagy that is critical for alpha-synuclein degradation and prevention of pathogenesis of Parkinson's disease. J. Neurosci. 35, 10613-10628 (2015).

53. Zhang, L. et al. Targeted methylation sequencing reveals dysregulated Wnt signaling in Parkinson disease. J. Genet. Genomics 43, 587-592 (2016).

54. Giacomello, M., Pyakurel, A., Glytsou, C. \& Scorrano, L. The cell biology of mitochondrial membrane dynamics. Nat. Rev. Mol. Cell Biol. 21, 204-224 (2020).

55. Wang, W., Ma, X., Zhou, L., Liu, J. \& Zhu, X. A conserved retromer sorting motif is essential for mitochondrial DLP1 recycling by VPS35 in Parkinson's disease model. Hum. Mol. Genet. 26, 781-789 (2017).

56. Filadi, R., Pendin, D. \& Pizzo, P. Mitofusin 2: from functions to disease. Cell Death Dis. 9,330 (2018).

57. Zhou, L., Wang, W., Hoppel, C., Liu, J. \& Zhu, X. Parkinson's disease-associated pathogenic VPS35 mutation causes complex I deficits. Biochim. Biophys. Acta Mol. Basis Dis. 1863, 2791-2795 (2017). 